\title{
Sistema de indicadores comparables de convivencia y seguridad ciudadana: un consenso de países ${ }^{1}$
}

\author{
Rodrigo Guerrero Velasco, ${ }^{2}$ María Isabel Gutiérrez Martínez, ${ }^{2}$ \\ Andrés Fandiño-Losada ${ }^{2}$ y Sonia Cardona²
}

Forma de citar Guerrero Velasco R, Gutiérrez Martínez MI, Fandiño-Losada A, Cardona S. Sistema de indicadores comparables de convivencia y seguridad ciudadana: un consenso de países. Rev Panam Salud Publica. 2012;31(3):253-9.

RESUMEN La región de las Américas, con una tasa de homicidios de 16,4 por 100000 habitantes (2004), es la segunda más violenta del mundo, pero la formulación de políticas públicas de prevención de violencia y delincuencia se ve obstaculizada por la ausencia de datos confiables y comparables sobre las diferentes manifestaciones del problema. El presente trabajo detalla un esfuerzo realizado por 14 países, patrocinado por el Banco Interamericano de Desarrollo y en asocio con otros organismos regionales, para desarrollar — de manera colectiva y consensuadaindicadores de convivencia y seguridad ciudadana que permitan comparaciones entre países, sistematizar métodos de producción y recolección de los datos pertinentes, generar politicas regionales y difundir e intercambiar buenas prácticas identificadas. Los indicadores escogidos fueron homicidio, muerte por lesiones de tránsito, suicidio, muerte por arma de fuego, delito sexual, violencia intrafamiliar, maltrato infantil, hurto, robo, hurto/robo de vehículos, secuestro, infracción de tránsito bajo efectos del alcohol, victimización general, victimización por robo, victimización por hurto, percepción de inseguridad, percepción de miedo, confianza en las instituciones y justificación del uso de violencia. Para cada indicador se acordó la forma de medición y las variables mínimas que deben tenerse en cuenta para contar con criterios de comparación apropiados.

Palabras clave Política social; seguridad; problemas sociales; violencia; indicadores de salud; sistemas de información; América Latina.

La violencia, la criminalidad y la inseguridad constituyen un problema de enorme magnitud que se extiende con rapidez en distintos lugares y ámbitos. Según estimaciones de la Organización

\footnotetext{
1 El presente estudio forma parte del proyecto "Sistema Regional de Indicadores Estandarizados de Convivencia y Seguridad Ciudadana (SES)", financiado por el Banco Interamericano de Desarrollo y llevado a cabo por el Instituto CISALVA de la Universidad del Valle (Cali, Colombia).

2 Instituto CISALVA. Universidad del Valle, Cali, Colombia. La correspondencia se debe dirigir a Rodrigo Guerrero Velasco. Correo electrónico: cisalva@univalle.edu.co
}

Mundial de la Salud (OMS) cada año más de 1,6 millones de personas pierden la vida de manera violenta en el mundo, de los cuales $51 \%$ corresponden a suicidios, $37 \%$ a homicidios y $11 \%$ a guerras y conflictos civiles. Este patrón es diferente en América Latina, donde las tasas de homicidios son casi tres veces mayores que las tasas de suicidios (1). Partiendo de datos nacionales disponibles en sus Países Miembros, la OMS ubica a la región de las Américas -16,4 homicidios por cada 100000 habitantes en
2004 — ${ }^{3}$ como la segunda más violenta del mundo, solo superada por África subsahariana $(2,3)$, si bien es cierto que en las Américas el número de homicidios incluye las muertes por guerras y conflictos civiles o armados.

En la Región la violencia alcanza proporciones epidémicas, constituyendo

\footnotetext{
La última referencia encontrada sobre homicidios consolidados para la región de las Américas fue publicada por la Organización Panamericana de la Salud (2). Con posterioridad, solo se encontraron datos individuales de diferentes países de la Región.
} 
una de las principales causas de muerte en el grupo etario de 15 a 44 años y resultando responsable de $15 \%$ de las defunciones de hombres y $7 \%$ de mujeres (4). De acuerdo con la OMS, solo en el año 2000 ocurrieron 199000 homicidios (92 por 100000 habitantes) en jóvenes de entre 10 y 29 años de edad (5). Y si bien es cierto que las altas tasas de homicidios son un fenómeno que afecta a todo el continente, también lo es que hay marcadas diferencias entre los países (6) (cuadro 1).

Por lo general, la información sobre violencia y criminalidad proviene de las agencias nacionales que recolectan datos con fines estadísticos, los cuales son procesados mediante diversos sistemas de información y publicados como boletines o anuarios, no siempre con la oportunidad que se requiere para el diseño de políticas públicas de preven-

CUADRO 1. Las 10 tasas (por 100000 habitantes) de homicidios más altas en la región de las Américas, 2007 y 2008

\begin{tabular}{llll}
\hline \multicolumn{1}{c}{ País } & \multicolumn{1}{c}{ Región } & Tasa & Año \\
\hline Honduras & América Central & 60,9 & 2008 \\
Jamaica & Caribe & 59,5 & 2008 \\
Venezuela & América del Sur & 52,0 & 2008 \\
El Salvador & América Central & 51,8 & 2008 \\
Guatemala & América Central & 45,2 & 2006 \\
Trinidad y & & & \\
$\quad$ Tabago & Caribe & 39,7 & 2008 \\
Colombia & América del Sur & 38,8 & 2007 \\
Saint Kitts y & & & \\
$\quad$ Nevis & Caribe & 35,2 & 2008 \\
Belice & América Central & 34,3 & 2008 \\
Anguila & Caribe & 27,6 & 2007 \\
\hline
\end{tabular}

Fuente: elaboración de los autores, con base en la referencia seis. ción y control y atención eficaces (7-11). Tal situación trae aparejadas variaciones substanciales según las agencias recolectoras de los datos, debidas fundamentalmente a que cada una recoge la información para sus propios fines y necesidades de acuerdo a su misión institucional (6, 11). Es así como las agencias de seguridad y justicia reúnen datos desde la perspectiva de la ley y de las medidas por adoptar, las de salud se centran en la naturaleza de la lesión y las de asistencia social se orientan al comportamiento del agresor y al sistema de atención a las víctimas $(12,13)$. En los distintos casos se identifican deficiencias importantes en materia de cobertura y/o integridad de la recopilación de los datos.

Por otra parte cuando se habla de seguridad muchas veces se utilizan conceptos distintos, razón por la cual los indicadores para estimarla no resultan comparables (14). Por ejemplo, si se toma el caso de los homicidios, que son relativamente fáciles de registrar debido a su gran impacto social, se pueden observar grandes diferencias en las cifras - para un mismo país y período- según la fuente, como se ilustra en el cuadro 2 con países de América Central $(9,15,16)$.

La ausencia de información comparable de buena calidad ha sido y sigue siendo un obstáculo para la formulación de políticas públicas regionales (17-19). Entendiendo que la violencia dificulta el desarrollo social y económico en América Latina y el Caribe, el Banco Interamericano de Desarrollo (BID) organizó en 2005 un Foro Interamericano sobre Seguridad Ciudadana en Medellín (Colombia) para identificar las posibles

CUADRO 2. Tasas de homicidios (por 100000 habitantes) en países de América Central, 2000-2006

\begin{tabular}{llcccc}
\hline Años & Fuentes & El Salvador & Guatemala & Honduras & Nicaragua \\
\hline 2000 & OEA & 37,3 & 25,8 & 49,9 & 9,0 \\
& PNUD & 45,0 & 28,0 & 0,0 & 9,0 \\
2001 & OEA & 34,6 & 25,2 & 53,7 & 10,0 \\
& PNUD & 40,0 & 30,0 & 0,0 & 10,0 \\
2002 & OEA & 31,1 & 30,7 & 55,9 & 10,0 \\
& PNUD & 39,0 & 32,0 & 69,0 & 10,0 \\
2003 & OEA & 32,7 & 35,0 & 33,6 & 12,0 \\
& PNUD & 40,0 & 37,0 & 65,0 & 12,0 \\
2004 & OEA & 41,0 & 36,3 & 31,9 & 12,0 \\
& PNUD & 49,0 & 38,0 & 35,0 & 12,0 \\
& OEA & 54,9 & 42,0 & 35,0 & 13,0 \\
2006 & PNUD & 62,0 & 44,0 & 37,0 & 13,0 \\
& OEA & 55,3 & 45,2 & 42,9 & 12,4 \\
& PNUD & 65,0 & 47,0 & 46,0 & 13,0 \\
\hline
\end{tabular}

Fuente: elaborado por los autores, con base en las referencias 15 y 16.

Nota: Abreviaturas. OEA: Organización de Estados Americanos; PNUD: Programa de las Naciones Unidas para el Desarrollo.

soluciones a este problema, al cual asistieron diversos funcionarios y expertos en desarrollo y convivencia ciudadana y los alcaldes de nueve ciudades de la región (Montevideo, São Paulo, Buenos Aires, San Pedro Sula, Río de Janeiro, Kingston, Bogotá, Medellín y Cali). En el marco de este foro y de las reuniones subsiguientes, participantes de Bolivia, Colombia, Ecuador, Honduras, Panamá, Perú y Venezuela se dieron a la tarea de construir una propuesta para mejorar la recolección de información, su calidad y el análisis de los datos y desarrollar un idioma común con el cual los países de la región pudieran adoptar un conjunto comparable de indicadores de violencia y convivencia ciudadana. De esta forma nació el proyecto - expuesto en el presente trabajo- Sistema Regional de Indicadores Estandarizados (Comparables) de Convivencia y Seguridad Ciudadana (SES).

Un indicador es una representación cuantitativa de la realidad que permite conocer en forma continua la situación de una determinada variable, medir su cambio en el tiempo y compararla con otras variables. Sirve para estimar el logro de objetivos, políticas, programas y proyectos y por tanto debe ser claro, relevante en relación al objeto de medición y estar disponible periódicamente (20-22).

El proyecto SES se inscribe dentro del programa para la promoción de Bienes Públicos Regionales del BID, instrumento de desarrollo que resalta la necesidad de apropiación de las funciones del programa por parte de los actores participantes (en este caso, las agencias $\mathrm{y}$ actores de la sociedad civil en cada uno de los países) así como su acción colectiva (23).

Una forma de lograr la comparabilidad entre los datos de diferentes países o sectores es utilizar sistemas de clasificación armonizados y aceptados en distintos países y contextos. La División de Estadísticas de las Naciones Unidas es quien se encarga de revisar, aprobar y registrar los sistemas de clasificación existentes. Por todo ello, las Clasificaciones de Referencia surgen de acuerdos internacionales y han sido aprobadas por la Comisión de Estadísticas de las Naciones Unidas (24). Entre estas clasificaciones se encuentran la Clasificación Industrial Internacional Estándar de las Actividades Económicas, la Clasificación Internacional Estándar de Ocupaciones, 
la Clasificación Internacional Estándar de la Educación y -específicamente en el sector salud- la Clasificación Estadística Internacional de Enfermedades y Problemas Relacionados con la Salud (CIE). La primera CIE fue adoptada en 1893 por el Instituto Internacional de Estadística y se ha venido actualizando periódicamente. Al momento está vigente la décima revisión (CIE-10), adoptada por la Asamblea Mundial de la Salud de la OMS en 1990, y actualmente se está trabajando en su revisión undécima (25).

Algunos de los aspectos que presentan las mayores dificultades para la producción estandarizada de información a partir de registros administrativos estatales son la escasa frecuencia de producción, los disímiles mecanismos y prioridades de registro en los niveles locales y la baja calidad de los datos (26). En este sentido, los objetivos del presente estudio son describir el método seguido en la selección, armonización y formulación de los indicadores del SES y presentar la versión final de los mismos. Un objetivo adicional es establecer un punto de partida para la construcción de la Clasificación Internacional de la Violencia y los Delitos (CIVD), siguiendo los principios que rigen las clasificaciones aceptadas internacionalmente y tomando como parámetro la CIE-10, con la cual existen algunas correspondencias (S00-T98 y V01-Y98).

\section{MATERIALES Y MÉTODOS}

Para la construcción de los Indicadores Estandarizados (Comparables) de Convivencia y Seguridad Ciudadana se siguió una metodología de revisión conceptual, diagnóstico situacional y concertación y consenso por parte de delegados nacionales de todos los países vinculados, contando también con el apoyo de expertos internacionales (18). En particular, se siguieron los siguientes pasos:

1. Identificación de las entidades nacionales responsables de la seguridad ciudadana y la convivencia.

2. Concertación sobre los conceptos de convivencia y seguridad ciudadana.

3. Establecimiento de las $\mathrm{UA}^{4}$ pertinentes a partir de los conceptos concertados.

\footnotetext{
4 Según el Departamento Administrativo Nacional de Estadísticas de Colombia (DANE), la UA se entiende "como el elemento mínimo de estudio, observable o medible, en relación con un conjunto de otros elementos que son de su mismo tipo" (20).
}

4. Priorización de los indicadores nacionales.

5. Identificación de las fuentes nacionales de información.

6. Construcción de las fichas técnicas de cada indicador, a partir de la experiencia del DANE (20).

Inicialmente, en cada país se convocó a que participaran las entidades nacionales responsables de la definición de políticas públicas y generación de información relacionada con la seguridad ciudadana y la convivencia, seleccionándose a una de ellas como coordinadora. A esta última se la denominó "subunidad técnica" y se la hizo responsable de tomar las decisiones técnicas del proyecto.

Para seleccionar los indicadores de convivencia y seguridad ciudadana, los investigadores del proyecto SES tuvieron en cuenta las estadísticas sobre violencia, delincuencia y conflictividad disponibles en los países participantes. También se hizo un esfuerzo para determinar la responsabilidad institucional y el método utilizado para la producción de datos nacionales, la cobertura de los sistemas de registro en cada país y la disponibilidad y oportunidad de acceso a los datos existentes.

Luego de haber realizado la construcción, identificación y priorización de los indicadores en cada país, se celebró en Cali un taller regional para seleccionar y estandarizar el conjunto de indicadores de convivencia y seguridad ciudadana, con base en los criterios de pertinencia, relevancia y disponibilidad $(21,22,27)$. Allí se reunieron funcionarios de distintas entidades de los países socios iniciales del proyecto ${ }^{5}$ - policía, institutos de medicina legal o forense, ministerios públicos y otros- junto a expertos de organismos regionales encargados de los temas abordados: la Organización Panamericana de la Salud, la Organización de Estados Americanos (OEA), la representación en Honduras del Programa de Naciones Unidas para el Desarrollo (PNUD), los Centros de Control de Enfermedades de los Estados Unidos y el Centro Nacional para la Prevención de Lesiones de México.

Posteriormente, con el objeto de lograr consensos sobre los conceptos, las variables y los flujos de transferencia de información aplicables a los indica-

Colombia, Ecuador, Honduras, Perú y República
Dominicana. dores, se realizaron seis mesas técnicas regionales en las cuales se analizó la factibilidad y la capacidad institucional para la producción de indicadores sobre los siguientes temas:

(i) Muertes violentas y en eventos de tránsito (terrestre) (Honduras, mayo de 2009).

(ii) Violencia intrafamiliar y delitos sexuales (Perú, junio de 2009).

(iii) Criminalidad (Colombia, agosto de 2009).

(iv) Victimización, percepción de inseguridad y convivencia (República Dominicana, noviembre de 2009).

(v) Convivencia y encuestas (Paraguay, abril de 2010).

(vi) Comité técnico sobre encuestas (Colombia, febrero de 2011).

Finalmente, y a partir de los acuerdos logrados y las conclusiones obtenidas en los talleres y mesas técnicas, se realizó la formulación final de los indicadores.

\section{RESULTADOS}

El consenso obtenido arrojó dos grandes categorías: la "seguridad ciudadana", definida como la situación de vivir en comunidad libre de riesgos, respetando los deberes y los derechos de todos los ciudadanos, y la "convivencia", entendida como la interrelación entre los ciudadanos y de estos con el Estado y el entorno público. Esta segunda categoría incluye la ausencia de violencia; la tolerancia entre las diversas opciones morales, culturales o sociales sin que se transgreda la ley; el cumplimiento de las reglas sociales, tanto formales como informales, y la simetría entre derechos y deberes.

Para ambas categorías se identificaron grandes temáticas a través de las cuales se pueden medir los fenómenos a abordar mediante los indicadores, las cuales denominamos unidades de análisis (UA), concepto relacionado con las categorías de análisis de la investigación cualitativa. Entre las UA se encuentran los homicidios, los suicidios, las denuncias por delitos sexuales y los hurtos. Una vez precisados los conceptos que definían estas UA, se formularon los indicadores que cumplían con los criterios de pertinencia, relevancia regional y disponibilidad de datos, conformándose así el conjunto de indicadores que serían obtenidos a través de los registros ad- 
ministrativos nacionales (12 indicadores [cuadro 3]).

También se establecieron las siguientes concordancias entre los indicadores regionales de convivencia y seguridad ciudadana y los códigos de la CIE-10:

- Homicidios: incluye las causas enumeradas en los códigos X85-Y09 y Y35-Y36 de la CIE-10. Se debe tener en cuenta que este indicador comprende homicidios por violencia interpersonal y homicidios por acciones legales y de guerra, los cuales aparecen como indicadores separados en otras partes del mundo.

- Muertes por lesiones de tránsito: incluye las causas de muerte por accidentes de transporte terrestre (V00V98). Sin embargo, no comprende accidentes de transporte en otros vehículos (V90-V99).

- Suicidios: incluye las causas de muerte enumeradas en los códigos X60-X84.
- Muertes por armas de fuego: incluye las causas enumeradas en los códigos W32-W34, X72-X74 y X93-X95.

- Maltrato infantil y de adolescentes: incluye las causas enumeradas en los códigos T74 y Y07. Se debe tener en cuenta que el código Y07 solo describe la relación entre víctima y victimario, la que debe complementarse con las lesiones descritas en los códigos X85Y09 de la CIE-10.

Para el caso de los indicadores que requieren de encuestas, se concluyó que ninguna de las encuestas disponibles, nacionales o regionales, cubría todos los ámbitos de interés. Como resultado adicional se estableció que las preguntas relacionadas con violencia sexual y violencia intrafamilar/familiar/doméstica deberían consignarse en cuestionarios separados de la encuesta de victimización general y percepción de seguridad, motivo por el cual se construyeron tres instrumentos, uno para cada uno de los temas propuestos.

Los expertos de los países participantes en las distintas mesas regionales establecieron los criterios mínimos para que los resultados de las encuestas sean comparables entre los países:

i) Selección de las muestras por métodos probabilísticos y con tamaño adecuado según la prevalencia estimada de la variable considerada y los márgenes de error aceptables.

ii) Preservación homogénea de los aspectos básicos de los cuestionarios, salvo ciertos modos de expresión propios de cada país, región o lugar.

iii) Períodos de referencia iguales o, al menos, que estén claramente identificados para permitir las comparaciones.

Después de varias sesiones de trabajo, se llegó de manera consensuada a las definiciones e indicadores sobre convi-

CUADRO 3. Indicadores regionales de convivencia y seguridad ciudadana para ser obtenidos a partir de registros administrativos en países de las Américas

\begin{tabular}{|c|c|c|}
\hline Unidad de análisis & Definición & Indicador \\
\hline Homicidio & $\begin{array}{l}\text { Muerte por lesión intencional ocasionada por otra persona. } \\
\text { Excluye muertes por lesiones de tránsito y otras lesiones no } \\
\text { intencionales. }\end{array}$ & 1. Tasa de homicidios por cada 100000 habitantes \\
\hline Muerte por lesiones de tránsito & $\begin{array}{l}\text { Muerte ocasionada por lesiones en un evento de tránsito } \\
\text { terrestre (o tráfico), sin importar el momento en que sucede } \\
\text { la muerte }\end{array}$ & 2. Tasa de muertes por tránsito por cada 100000 habitantes \\
\hline Suicidio & $\begin{array}{l}\text { Muerte causada por lesión intencional autoinfligida, en } \\
\text { personas mayores de } 5 \text { años }\end{array}$ & $\begin{array}{l}\text { 3. Tasa de suicidios por cada } 100000 \text { habitantes mayores de } \\
5 \text { años }\end{array}$ \\
\hline Muerte por armas de fuego & $\begin{array}{l}\text { Muerte causada por lesiones de proyectil disparado con arma } \\
\text { de fuego, sin importar la intencionalidad del evento }\end{array}$ & $\begin{array}{l}\text { 4. Tasa de muertes por armas de fuego por cada } 100000 \\
\text { habitantes }\end{array}$ \\
\hline Delito sexual & $\begin{array}{l}\text { Cualquier acto de carácter sexual que se realice sin el } \\
\text { consentimiento de una persona, haya o no penetración. } \\
\text { También en el caso de niños, niñas y adolescentes, con o sin } \\
\text { su consentimiento }\end{array}$ & $\begin{array}{l}\text { 5. Tasa de denuncias de delitos sexuales por cada } 100000 \\
\text { habitantes }\end{array}$ \\
\hline $\begin{array}{l}\text { Violencia intrafamiliar/familiar/ } \\
\text { doméstica }\end{array}$ & $\begin{array}{l}\text { Todo acto u omisión que ocasione algún daño entre los } \\
\text { miembros de la familia o pareja/ex-pareja. Excluye los delitos } \\
\text { sexuales }\end{array}$ & $\begin{array}{l}\text { 6. Tasa de denuncias de violencia intrafamiliar/familiar/ } \\
\text { doméstica por cada } 100000 \text { habitantes }\end{array}$ \\
\hline $\begin{array}{l}\text { Maltrato infantil y de } \\
\text { adolescentes }\end{array}$ & $\begin{array}{l}\text { Cualquier acción u omisión causada por instituciones o } \\
\text { por personas con quien exista una relación, que vulnere } \\
\text { la integridad física o psicológica de los niños, niñas y } \\
\text { adolescentes. Excluye los delitos sexuales }\end{array}$ & $\begin{array}{l}\text { 7. Tasa de denuncias de maltrato infantil y de adolescentes por } \\
\text { cada } 1000 \text { menores de } 18 \text { años }\end{array}$ \\
\hline Hurto & $\begin{array}{l}\text { Apoderarse de un bien ajeno sin ejercicio de violencia ni } \\
\text { fuerza sobre las personas o las cosas }\end{array}$ & 8. Tasa de denuncias de hurto por cada 100000 habitantes \\
\hline Robo & $\begin{array}{l}\text { Apoderarse de un bien ajeno mediante el ejercicio de } \\
\text { cualquier tipo de violencia sobre las personas y/o cosas }\end{array}$ & 9. Tasa de denuncias de robo por cada 100000 habitantes \\
\hline Hurto y robo de automotor & Apoderarse de un automotor, con o sin violencia & $\begin{array}{l}\text { 10. Tasa de denuncias de robo y hurto por cada } 10000 \\
\text { automotores matriculados }\end{array}$ \\
\hline Secuestro & $\begin{array}{l}\text { Privación de la libertad de una persona plenamente } \\
\text { identificada, en contra de su voluntad, exigiendo una ventaja } \\
\text { económica o de otra índole a cambio de su liberación }\end{array}$ & 11. Tasa de denuncias de secuestro por cada 100000 habitantes \\
\hline $\begin{array}{l}\text { Infracción de normas de } \\
\text { tránsito por conducir en estado } \\
\text { de embriaguez etílica }\end{array}$ & $\begin{array}{l}\text { Transgresión a las normas de seguridad vial por conducir con } \\
\text { las capacidades físicas disminuidas debido al consumo de } \\
\text { alcohol }\end{array}$ & $\begin{array}{l}\text { 12. Tasa de infracciones por conducir en estado de embriaguez } \\
\text { etílica en mayores de } 15 \text { años por cada } 100000 \text { habitantes } \\
\text { mayores de } 15 \text { años }\end{array}$ \\
\hline
\end{tabular}

Fuente: elaborado por los autores. 
vencia y seguridad ciudadana que pueden obtenerse a través de encuestas (10 indicadores [cuadro 4]).

\section{DISCUSIÓN}

El SES puso de manifiesto que todos los países realizan esfuerzos por contar con información que facilite la toma de decisiones, pero se apoyan en datos de diferentes fuentes que recogen información según sus propias necesidades misionales. Con frecuencia la recolección se hace a través de formatos que suministran datos agregados para períodos específicos. Pocas instituciones cuentan con la tecnología necesaria para la extracción y desagregación de la información y muchas menos con sistemas de geo-referenciación. Hay brechas importantes en los datos sobre violencia en cada país, lo que dificulta la toma de decisiones adecuadas y oportunas en materia de políticas públicas.

Por esta razón, uno de los aspectos abordados en el SES desde el comienzo fue la necesidad de utilizar métodos aceptados internacionalmente en los procesos de aseguramiento y control de calidad de los sistemas de información, para resolver los problemas existentes en la recolección, captura, sistematización, clasificación, ordenamiento y análisis de los datos $-\mathrm{y}$ siguiendo los principios de oportunidad, exactitud, consistencia e integridad $(9,21,22)$. Desde el principio fue patente la necesidad de utilizar tasas estandarizadas para eliminar el efecto de la composición diversa de las poblaciones. Así, el interés por disponer de datos comparables sobre seguridad y convivencia resultó evidente cuando se incorporaron al proyecto SES los nuevos países participantes, que pasaron de los cuatro iniciales -Colombia, Ecuador, Honduras y Perú - a un total de 14 países en 2011 —sumando a Paraguay, República Dominicana, Uruguay, Chile, Costa Rica, El Salvador, Guyana, Jamaica, México y Nicaragua-, además de dos ciudades capitales, Quito y Buenos Aires.

Fue relativamente sencillo identificar los indicadores relativos a la violencia, pues se adoptó la definición de violencia interpersonal de la OMS, sin especificar si se trata o no de violencia colectiva (28). La situación fue más compleja para los casos de la seguridad ciudadana y de la convivencia, pues no existen definicio- nes que tengan una aceptación universal. La seguridad ciudadana se definió como la situación de vivir en comunidad libre de riesgos, respetando los deberes y los derechos de todos los ciudadanos y es claramente diferente de la seguridad humana, que incluye además aspectos del desarrollo humano $(16,29,30)$.

La mayoría de los países no contaba con experiencia en el manejo de información sobre convivencia, por lo que el proceso de selección de estos indicadores fue lento y difícil. Finalmente, la convivencia se definió como la interrelación entre los ciudadanos, y de estos con el Estado y el entorno público. Para diseñar esta última categoría de indicadores se tuvo en cuenta la experiencia de la Alcaldía de Bogotá en 2001-2003, durante la cual se desarrolló un instrumento de recolección de información sobre convivencia y de evaluación de las estrategias pedagógicas para su promoción $(31,32)$.

Los datos correspondientes a violencia intrafamiliar/familiar/doméstica se recolectan a través de diversas fuentes institucionales según los ordenamientos jurídico y social de cada país, pero no existen mecanismos adecuados para la producción de información desagre-

CUADRO 4. Indicadores regionales de convivencia y seguridad ciudadana para ser obtenidos a partir de encuestas poblacionales en países de las Américas

\begin{tabular}{|c|c|c|}
\hline Unidad de análisis & Definición & Indicador \\
\hline Violencia sexual & $\begin{array}{l}\text { Todo acto sexual, la tentativa de consumar un acto sexual, } \\
\text { los comentarios o insinuaciones sexuales no deseados, } \\
\text { o las acciones para comercializar o utilizar de cualquier } \\
\text { modo la sexualidad de una persona mediante coacción, } \\
\text { independientemente de la relación entre el agresor y la } \\
\text { víctima, en cualquier ámbito, incluidos los hogares o lugares } \\
\text { de trabajo }\end{array}$ & 1. Prevalencia de violencia sexual \\
\hline $\begin{array}{l}\text { Violencia intrafamiliar/familiar/ } \\
\text { doméstica }\end{array}$ & $\begin{array}{l}\text { Todo acto u omisión que ocasione algún daño entre los } \\
\text { miembros de la familia o pareja/ex-pareja. Excluye los delitos } \\
\text { sexuales }\end{array}$ & 2. Prevalencia de violencia intrafamiliar/familiar/doméstica \\
\hline Victimización delictiva & $\begin{array}{l}\text { Proceso en el que una persona sufre las consecuencias de un } \\
\text { delito, cometido por otra persona o por una institución }\end{array}$ & $\begin{array}{l}\text { 3. Porcentaje de victimización delictiva en mayores de } 18 \text { años } \\
\text { 4. Porcentaje de victimización por robo en mayores de } 18 \text { años } \\
\text { 5. Porcentaje de victimización por hurto en mayores de } 18 \text { años }\end{array}$ \\
\hline Percepción de inseguridad & Sensación de desprotección general asociada con el delito & $\begin{array}{l}\text { 6. Porcentaje de personas con percepción de inseguridad en } \\
\text { mayores de } 18 \text { años }\end{array}$ \\
\hline Percepción de riesgo & $\begin{array}{l}\text { Sensación asociada con la posibilidad de ser víctima de un } \\
\text { acto delictivo }\end{array}$ & $\begin{array}{l}\text { 7. Porcentaje de personas con percepción de riesgo en mayores } \\
\text { de } 18 \text { años }\end{array}$ \\
\hline Percepción de miedo & $\begin{array}{l}\text { Cambios en las actitudes o comportamientos de las personas } \\
\text { ante la probabilidad de ser víctimas de algún futuro delito }\end{array}$ & $\begin{array}{l}\text { 8. Porcentaje de personas con percepción de miedo en mayores } \\
\text { de } 18 \text { años }\end{array}$ \\
\hline Actitudes hacia la violencia & $\begin{array}{l}\text { Proceso subjetivo de validación o rechazo frente a las } \\
\text { diversas formas de daño sobre personas, grupos, objetos o } \\
\text { ideas }\end{array}$ & $\begin{array}{l}\text { 9. Porcentaje de personas que justifican el uso de violencia en } \\
\text { mayores de } 18 \text { años }\end{array}$ \\
\hline Confianza en las instituciones & $\begin{array}{l}\text { Es el grado de credibilidad que tienen las instituciones } \\
\text { encargadas de prevenir y proteger a la población, investigar } \\
\text { los hechos delictivos ocurridos, sancionar a sus causantes y } \\
\text { reinsertarlos en la sociedad }\end{array}$ & $\begin{array}{l}\text { 10. Porcentaje de personas con confianza en las instituciones en } \\
\text { mayores de } 18 \text { años }\end{array}$ \\
\hline
\end{tabular}

Fuente: elaborado por los autores. 
gada, pese a los compromisos internacionales asumidos para recabar este tipo de datos $(33,34)$. Por esa razón se optó por recoger los datos correspondientes a denuncias por violencia intrafamiliar/ familiar/doméstica; sin embargo, y debido al alto porcentaje de sub-registro en esta materia, se recomendó complementar esos datos con otros provenientes de las encuestas especializadas aquí indicadas.

Adicionalmente, durante el desarrollo y formulación de los indicadores propuestos, se tuvieron en cuenta las concordancias con la CIE-10 para apoyar de ese modo el cálculo de los indicadores con estadísticas provenientes del sector salud y de la medicina forense. No obstante, los códigos de la CIE-10 no brindan ciertos detalles requeridos para algunos de los indicadores, por ejemplo el código Y07.8 se refiere a "otros síndromes de maltrato por otra persona especificada" sin distinguir si esa persona es un familiar o no, cuestión que resulta indispensable para el indicador de violencia doméstica.

Como ya ha sido planteado, para que la cadena de producción de información funcione apropiadamente es necesario realizar una capacitación cuidadosa tanto de los funcionarios que hacen el primer registro de los datos como de quienes participan en su control de calidad y consolidación. Sólo así las decisiones en materia de políticas públicas podrán basarse en datos oportunos, válidos, íntegros y exactos.

Así y todo, para obtener estadísticas comparables se requiere no solo de estos aspectos técnicos propios de los indicadores sino también de la voluntad política de los Estados, quienes deben adoptar definiciones homogéneas para las diferentes formas de la violencia y los delitos. Es evidente que dichas estadísticas se encuentran al final de un proceso extremadamente complejo, pues las definiciones de los delitos y la violencia se basan en el sistema legal de cada país y se relacionan estrechamente con temas muy sensibles de las políticas nacionales - por esta razón se acordó que fueran los propios países quienes difundieran la información obtenida.

\section{Conclusiones}

El primer paso para abordar el análisis de la violencia y la criminalidad es establecer definiciones comunes para todos los países, lo que permitirá orientar los mecanismos y las intervenciones destinados a enfrentarlas desde políticas públicas basadas en evidencias. El respaldo e involucramiento de los organismos correspondientes de las Naciones Unidas (Oficina de las Naciones Unidas contra la Droga y el Crimen [UNODC, por sus siglas en inglés] y PNUD) y de organizaciones regionales (BID, OEA y el Sistema Integrado de Países Centro Americanos [SICA]) ha sido de gran importancia para afianzar los logros de este trabajo. Adicionalmente, se debe tener en cuenta que el crimen ha rebasado las fronteras de los países para convertirse en un fenómeno transnacional, requiriéndose de la armonización de sus definiciones para lograr su control efectivo, razón por la cual se ha planteado el desarrollo y formulación de la $\operatorname{CIVD}(6,9,15)$.

Vale destacar que el resultado fundamental de este proceso ha sido lograr acuerdos básicos sobre los conceptos de convivencia y seguridad ciudadana, como punto de partida para el desarrollo de iniciativas que promuevan la acción de los gobiernos nacionales y de los organismos internacionales para estandarizar los lineamientos de calidad para el registro, procesamiento y análisis de la información. Estos acuerdos ya han sido alineados con la CIE-10 para los temas en común y con las directrices de otros organismos multilaterales, como UNODC y OEA, para afianzar su difusión y aplicación.

Se espera que este esfuerzo ayude a consolidar un sistema de indicadores estandarizados de convivencia y seguridad ciudadana para la Región, que proporcione a los gobiernos una herramienta confiable que oriente las intervenciones destinadas a combatir la inseguridad, el crimen y la violencia, y facilite la evaluación de las acciones de prevención y control, así como el diseño coordinado de políticas regionales eficaces.

Agradecimientos. Los autores desean expresar su gratitud a los coordinadores de las subunidades técnicas de los países e instituciones que las conforman. Agradecen también la participación de María Carolina Franco y Paola Margarita Buendía (Departamento Nacional de Planeación de Colombia), Laura Bocalandro y Rafael Villa (programa de Bienes Públicos Regionales [BID]) y Jorge Lamas y Jorge Srur (BID). También reconocen la valiosa ayuda de Rafael Espinosa del Vallín en la consolidación de la propuesta, y de Jorge Mena, Yamileth Ortiz, Iván Darío Nungo y Juan Pablo Gordillo, responsables técnicos del proyecto. Agradecen asimismo la participación del personal administrativo del proyecto con sede en Cali y en las oficinas del BID en los países, de los expertos y otros colegas que aportaron al proyecto.

\section{REFERENCIAS}

1. World Health Organization (WHO). Regional burden of disease estimates for 2004. Disponible en: http://www.who.int/healthinfo/ global_burden_disease/estimates_regional/ en/index.html Acceso el 5 de enero de 2012.

2. Pan American Health Organization (PAHO). Health Information and Analysis Project. Health Situation in the Americas: Basic Indicators, 2009. Washington, D.C.: PAHO; 2009.

3. Alda E, Béliz G. ¿Cuál es la salida? La agenda inconclusa de la seguridad ciudadana. Washington, D.C.: Banco Interamericano de Desarollo; 2007.
4. Dahlbergh LL, Krug EG. Violence - a global public health problem. In: Krug EG, Dahlbergh LL, Mercy JA, Zwi A, Lozano R, eds. World Report on Violence and Health. Geneva: World Health Organization; 2002.

5. Mercy JA, Butchart A, Farrington D, Cerdá M. Youth Violence. In: Krug EG, Dahlbergh LL, Mercy JA, Zwi A, Lozano R, eds. World Report on Violence and Health. Geneva: World Health Organization; 2002.

6. United Nations Office on Drugs and Crime (UNODC). 2011 Global Study on Homicide. Trends, Contexts, Data. Vienna: UNODC; 2011.
7. Dammert L, Ruz F, Salazar F. ¿Políticas de seguridad a ciegas? Desafíos para la construcción de sistemas de información en América Latina. Santiago de Chile: FLACSO-Chile; 2008.

8. International Centre for the Prevention of Crime. International Report on Crime Prevention and Community Safety. Montréal: International Centre for the Prevention of Crime; 2008.

9. Harrendorf S, Heiskanen M, Malby S. International Statistics on Crime and Justice. European Institute for Crime Prevention and Control, Affiliated with the United Nations 
(HEUNI) and United Nations Office on Drugs and Crime (UNODC). HEUNI Publication Series No. 64. Helsinki: HEUNI; 2010.

10. Fondo Monetario Internacional (FMI). Marco para Evaluar la Calidad de las Estadísticas (MECAD) de Finanzas Públicas. Washington D.C.: FMI; 2003

11. Gutierrez-Martinez MI, Espinosa del Vallín $\mathrm{R}$, Fandino A, Oliver RL. The evaluation of a surveillance system for violent and non-intentional injury mortality in Colombian cities. Int J Inj Contr Saf Promot. 2007;14(2):77-84.

12. Dammert L, Salazar F, Montt C, González P. Crimen e inseguridad. Indicadores para las Américas. Proyecto Violencia y criminalidad en América Latina y el Caribe. Santiago de Chile: FLACSO-Chile - BID; 2010.

13. Carrión F, Espín J. Un lenguaje colectivo en construcción: El diagnóstico de la violencia. Quito: FLACSO-Ecuador - ICLEI - Alcaldía Metropolitana de Quito; 2009.

14. Ramacciotti B. Democracy and Multidimensional Security: The rising need for citizen security in Latin America. Paper presented at: Seminar on "Security and Democratic Governability: Addressing Challenges in Latin America"; 2005 Mar 15; Washinton, DC.

15. Organización de los Estados Americanos (OEA). La seguridad pública en las Américas: retos y oportunidades. 2. ${ }^{\mathrm{a}}$ ed. Washington, D.C.: OEA; 2008.

16. Programa de las Naciones Unidas para el Desarrollo (PNUD). Abrir espacios para la seguridad ciudadana y el desarrollo humano. Informe sobre desarrollo humano para América Central 2009-2010. Bogotá: PNUD; 2009.

17. Guerrero R. Sistemas de información en los programas de prevención de violencia. En: Alda E, Béliz G, eds. ¿Cuál es la salida? La agenda inconclusa de la seguridad ciudadana.
Washington, D.C.: Banco Interamericano de Desarrollo; 2007

18. Gutierrez-Martinez MI, Espinosa del Vallín R. Guía metodológica para la replicación de observatorios municipales de violencia. Cali: CATORSE SCS; 2008.

19. Weitzman BC, Silver D, Brazill C. Efforts to Improve Public Policy and Programs through Data Practice: Experiences in 15 Distressed American Cities. Public Administration Review. 2006;66(3):1540-6210.

20. Departamento Administrativo Nacional de Estadísticas (DANE), Colombia. Herramientas estadísticas para una gestión territorial más efectiva. Guía para diseño, construcción e interpretación de indicadores. Bogotá: DANE; 2009.

21. Concha-Eastman A, Villaveces A. Guías para el diseño, implementación y evaluación de sistemas de vigilancia epidemiológica de violencia y lesiones. Washington, D.C.: Organización Panamericana de la Salud; 2001.

22. Lippeveld T, Sauerborn, Bodart C. Design and implementation of health information systems. Geneva: World Health Organization; 2000.

23. Bocalandro L, Villa R. Bienes públicos regionales: promoviendo soluciones innovadoras en Latinoamérica y el Caribe. Washington D.C: Banco Interamericano de Desarrollo; 2009.

24. Chamie M. Preamble: Family of International Economic and Social Classifications. In: Fourth Meeting of the Expert Group on International Economic and Social Classifications [New York, 2-4 Nov 1998]. New York: United Nations Statistics Division; 1998.

25. World Health Organization (WHO). The International Statistical Classification of Diseases and Related Health Problems, Tenth revision (Vol. 2). Geneva: WHO; 1993.
26. Aspinall P. Rapid Scoping Study. Canterbury: University of Kent; 2010.

27. Holder Y, Peden M, Krug E, Lund J, Gururaj $\mathrm{G}$, Kobusingye $\mathrm{O}$. Injury surveillance guidelines. Geneva: World Health Organization; 2001

28. Krug EG, Dahlbert L, Mercy J, Swi A, Lozano R. World Health Report on Violence. Geneva: World Health Organization; 1996.

29. Kjaerulf F, Barahona R. Preventing violence and reinforcing human security: a rightsbased framework for top-down and bottomup action. Rev Panam Salud Publica. 2010; 27(5):382-95.

30. United Nations Development Programme (UNDP). Human Development Report 1994. New York: UNDP; 1994

31. Sánchez E, Castro C. Cultura ciudadana en Bogotá: nuevas perspectivas. Bogotá: Cámara de Comercio/Secretaría de cultura, recreación y deporte/Fundación Terpel/Corpovisionarios; 2009.

32. Mockus A, Corzo J. Cumplir para convivir. Factores de convivencia y su relación con normas y acuerdos. Bogotá: Universidad Nacional de Colombia/Instituto de Estudios Políticos y Relaciones Internacionales; 2003.

33. United Nations. Manual for the Development of a System of Criminal Justice Statistics. New York: United Nations; 2003.

34. World Bank. World Development Report 2011: Conflict, Security, and Development. Washington D.C.: World Bank; 2011.

Manuscrito recibido el 12 de julio de 2011. Aceptado para publicación, tras revisión, el 28 de diciembre de 2011.
ABSTRACT

System of comparable indicators for peaceful coexistence and citizen security: a consensus among countries

Key words
The Region of the Americas, with a homicide rate of 16.4 per 100,000 population (2004), is the second most violent region in the world, but public policy-making for the prevention of violence and crime is hampered by the absence of reliable and comparable data on the problem's various manifestations. This paper describes a 14-country initiative, sponsored by the Inter-American Development Bank in partnership with other regional agencies, to develop-collectively and by consensusindicators for peaceful coexistence and citizen security that permit comparisons among countries, systematization of methods for the production and collection of pertinent data, the development of regional policies, and the dissemination and sharing of good practices. The selected indicators were homicide, death from road traffic injuries, suicide, death by firearm, sexual crime, family violence, child abuse, robbery, theft, vehicle theft and robbery, abduction, traffic offenses committed while under the influence of alcohol, general victimization, victimization by theft, victimization by robbery, perception of insecurity, perception of fear, confidence in institutions, and justification of the use of violence. For each indicator, agreement was reached on the type of measurement and the minimum variables that should be taken into account to have appropriate criteria for comparison.

Public policy; safety; social problems; violence; health status indicators; information systems; Latin America. 